\title{
Medicación prehospitalaria en pacientes hospitalizados por COVID-19 en un hospital público de Lima-Perú
}

\section{Pre-hospitalary medication in COVID-19 patients from a public hospital in Lima-Peru}

\author{
Correspondencia \\ Ernesto Zavala-Flores \\ ernestozav123@gmail.com \\ Recibido: 07/08/2020 \\ Aprobado: 20/08/2020 \\ Citar como: Zavala-Flores \\ E, Salcedo-Matienzo J. \\ Medicación prehospitalaria \\ en pacientes hospitalizados \\ por COVID-19 en un hospital \\ público de Lima-Perú. Acta \\ Med Peru. 2020;37(3):393-5. \\ doi: https://doi.org/10.35663/ \\ amp.2020.373.1277
}

\section{Ernesto Zavala-Flores ${ }^{1,2, a}$, Jannin Salcedo-Matienzo ${ }^{2, a}$}

Hospital Cayetano Heredia. Lima, Perú

Universidad Peruana Cayetano Heredia. Lima, Perú.

Médico cirujano.

\section{Señor editor,}

EI COVID-19 es la nueva enfermedad que en cuestión de meses se ha esparcido por más de 140 países. Actualmente, se ha vuelto un problema para la salud pública y un desafío para la comunidad médica debido a la falta de un tratamiento específico y/o vacuna ${ }^{[1]}$.

Esto ha generado que en varios países se elaboren protocolos de tratamiento para esta enfermedad en base a medicamentos cuya eficacia aún no ha sido demostrada; así como a la práctica de la automedicación, la cual se define como el uso de algún medicamento sin previa consulta con un médico para tratar algún síntoma o enfermedad ${ }^{\left[{ }^{[2]}\right.}$. Este estudio tiene como objetivo determinar la frecuencia de pacientes que recibieron algún tipo de medicación para el COVID-19 previo a su hospitalización.

Se estudiaron pacientes hospitalizados con diagnóstico de COVID-19 (confirmado o sospechoso), en el Hospital Cayetano Heredia, en Lima, durante la tercera semana del mes de junio del 2020 (del 14 al 21 de junio). Se incluyeron pacientes hospitalizados con diagnóstico de COVID-19 (confirmados o sospechosos) mayores de 18 años. Los criterios de exclusión fueron: pacientes hospitalizados cuyo diagnóstico de COVID-19 fuese un hallazgo laboratorial sin sintomatología respiratoria.

De un total de 132 pacientes hospitalizados con una media de edad de 60,3 años. 120 pacientes presentaron diagnóstico confirmado para COVID-19 con prueba rápida y/o prueba molecular (PCR), el sexo masculino fue el predominante $(69,7 \%)$ con una relación de 2,3 a 1 con respecto al sexo femenino, y la media de tiempo de enfermedad previo a la admisión hospitalaria fue de 8,0 $\pm 3,7$ días.

59 pacientes $(44,7 \%)$ presentaron al menos una comorbilidad; y 19 de ellos, tuvieron dos o más comorbilidades. La más frecuente fue la hipertensión arterial $(23,5 \%)$, y la dualidad más frecuente, diabetes tipo 2 e hipertensión arterial. 127 pacientes $(96,2 \%)$ recibieron soporte oxigenatorio; de ellos, 25 estuvieron con vía cánula binasal (18,9\%); 92, mascara reservorio (69,7\%); y 10, ventilación mecánica invasiva $(7,6 \%)$.

Del total, 106 pacientes (80,3\%) usaron fármacos antes de la admisión hospitalaria; de los cuales, 36 $(33,9 \%)$ se automedicaron. 97 pacientes $(91,5 \%)$ usaron dos o más medicamentos, siendo los antibióticos los más frecuentes $(85,8 \%)$, seguido por la ivermectina (66,9\%). Dentro del grupo de medicamentos $\sin$ prescripción médica, los más frecuentes fueron los antibióticos (28,3\%) (Tabla 1).

El promedio de los pacientes infectados por COVID-19 en nuestro país oscila entre los 60-70 años, rango que coincide con la media de 60,3 años encontrada en la población del presente estudio ${ }^{[3]}$. 
Tabla 1. Medicación usada por pacientes con COVID-19 antes de la admisión hospitalaria.

\begin{tabular}{lccc}
\multicolumn{1}{c}{ Medicamentos } & $\begin{array}{c}\text { Número de pacientes } \\
\mathbf{n}(\%)\end{array}$ & $\begin{array}{c}\text { Con prescripción médica } \\
\mathbf{n}(\%)\end{array}$ & $\begin{array}{c}\text { Sin prescripción médica } \\
\mathbf{n}(\%)\end{array}$ \\
\hline Antibióticos & $91(85,8)$ & $61(57,5)$ & $30(28,3)$ \\
Azitromicina & 66 & 52 & 14 \\
Ceftriaxona & 21 & 18 & 3 \\
Amoxicilina & 11 & 4 & 7 \\
Claritromicina & 5 & 4 & 1 \\
Levofloxacino & 4 & 4 & 0 \\
Cefuroxima & 2 & 1 & 1 \\
TMP/SMX & 2 & 1 & 1 \\
Otros & 5 & 5 & 0 \\
Ivermectina & $71(66,9)$ & $49(46,2)$ & $22(20,7)$ \\
Corticoides & $58(54,7)$ & $40(37,7)$ & $18(17,0)$ \\
Prednisona & 29 & 18 & 11 \\
Dexametasona & 38 & 28 & 10 \\
Paracetamol & $35(33,1)$ & $22(20,8)$ & $13(12,3)$ \\
Aspirina & $17(16,0)$ & $12(11,3)$ & $5(4,7)$ \\
Hidroxicloroquina & $11(10,3)$ & $10(9,4)$ & $1(0,9)$ \\
AINES & $17(16,0)$ & $12(11,3)$ & $5(4,7)$ \\
Enoxaparina & $4(3,8)$ & $4(3,8)$ & 0 \\
Otros & $20(18,8)$ & $18(16,9)$ & $2(1,9)$ \\
\hline
\end{tabular}

* 97 pacientes utilizaron dos o más medicamentos como tratamiento ambulatorio para la infección por COVID-19 previo a la admisión hospitalaria.

Por otro lado, a la fecha, la infección por SARS-CoV-2 no tiene un tratamiento específico ni preventivo. Diversos estudios in vitro postulan que algunos medicamentos tendrían un posible efecto sobre la replicación viral; sin embargo, aún no se ha demostrado este beneficio en los estudios clínicos ${ }^{[1,4,5]}$.

Esta incertidumbre en el tratamiento ha originado que se usen medicamentos por «plausibilidad fisiopatológica» o «efectos in vitro» alegando un posible efecto sobre la enfermedad ${ }^{[1,5]}$. Esto ha llevado a que algunos profesionales de la salud prescriban medicamentos sin eficacia demostrada a los pacientes y esto, junto a la dificultad para el acceso a la salud, ha contribuido a que los mismos opten por la automedicación.

En el presente estudio se encontró que hasta el $80,0 \%$ de los pacientes hospitalizados por COVID-19 recibieron algún tipo de tratamiento previo a la admisión hospitalaria y el 33,9\% lo hizo sin una prescripción médica (automedicación). Los antibióticos fueron los medicamentos más usados por los pacientes previo a la hospitalización $(85,8 \%)$, siendo la azitromicina el más usado $(62,2 \%)$, con o sin prescripción médica, seguido por otros antibióticos como la ceftriaxona, amoxicilina y claritromicina. Esto difiere de lo encontrado por Hermoza et al. (2016), quienes encontraron que el grupo de fármacos más frecuentemente usados como automedicación en un distrito de Lima-Perú fueron los AINES, antigripales y fármacos gastrointestinales ${ }^{[6]}$. Esta diferencia recaería en el contexto actual de la pandemia y la serie de tratamientos que en algún momento se postularon como «opciones terapéuticas» para el COVID-19.

Del mismo modo, en nuestra serie se encontró que los pacientes usaron otros medicamentos con o sin indicación médica, como la ivermectina $(66,9 \%)$, corticoterapia $(54,7 \%)$, o hidroxicloroquina (10,3\%). Estos medicamentos, al igual que la azitromicina, no tienen evidencia científica que permita aseverar que su uso impacte favorablemente en el curso de la enfermedad. Mientras que, a la fecha, el único medicamento que ha mostrado beneficios al disminuir la mortalidad en pacientes hospitalizados que requieran soporte oxigenatorio es la dexametasona, como lo reportan en el ensayo clínico RECOVERY ${ }^{[7]}$.

Es importante tener en consideración que la prescripción de estos medicamentos lejos de beneficiar al paciente, lo que podrían originar es elevar el riesgo de reacciones adversas medicamentosas, aumentar la resistencia antibiótica (en el caso de los antibióticos), así como retrasar la llegada de los pacientes al ambiente hospitalario ${ }^{[8-10]}$. Adicionalmente, en el presente estudio se encontró que la media entre el inicio de los síntomas y la admisión hospitalaria fue de 8 días; lo que podría 
explicarse por el hecho de que muchos pacientes esperan a que los medicamentos usados previamente hagan algún efecto; lo que, en casos de infección moderada-severa, podría contribuir a la mortalidad de estos pacientes debido a que no recibirían oportunamente las medidas de soporte necesarias.

Esta pandemia ha develado una serie de dificultades en diversos ámbitos de la salud, entre ellos la prescripción de medicamentos sin eficacia demostrada contra la infección por SARS-CoV-2 (por una parte del sector salud). Así mismo, en la población estudiada se encontró una alta proporción de pacientes que se automedicaron con alguno de estos fármacos.

Se sugiere fortalecer las estrategias educativas para la población en aras de mitigar la automedicación, así como los efectos adversos que conllevaría esta práctica. Del mismo modo, mantener una conducta vigilante sobre los profesionales de la salud que hacen promoción, en los medios de comunicación, del uso de medicamentos sin evidencia científica para el tratamiento del COVID-19.

Contribuciones de autoría: Los autores participaron en el diseño de estudio, recolección de información, interpretación y discusión de los resultados, redacción del manuscrito y evaluación de la versión final.

Potenciales conflictos de interés: Los autores declaran no tener conflictos de interés.

Fuente de financiamiento: Autofinanciado

\section{ORCID}

Ernesto Zavala-Flores, https://orcid.org/0000-0002-7389-5576 Jannin Salcedo-Matienzo, https://orcid.org/0000-0002-92213040

\section{REFERENCIAS BIBLIOGRÁFICAS}

1. Wong A. COVID-19 and toxicity from potential treatments: Panacea or poison. Emerg Med Australas. 2020;32(4):697-9. doi:10.1111/1742-6723.13537

2. Fereidouni Z, Kameli Morandini M, Najafi Kalyani M. Experiences of self-medication among people: a qualitative meta-synthesis. Daru J Fac Pharm Tehran Univ Med Sci. 2019;27(1):83-9. doi:10.1007/ s40199-019-00244-9

3. Ministerio de Salud del Perú. COVID-19 en el Perú [Internet]. Lima, Perú: MINSA; 2020 [citado 27 de junio de 2020]. Disponible en: https://covid19.minsa.gob.pe/

4. Gbinigie K, Frie K. Should azithromycin be used to treat COVID-19? A rapid review. BJGP Open. 2020;4(2). doi:10.3399/ BJGPOPEN20X101094

5. Jean S-S, Lee P-I, Hsueh P-R. Treatment options for COVID-19: The reality and challenges. J Microbiol Immunol Infect. 2020;53(3):43643. doi:10.1016/J.JMII.2020.03.034

6. Hermoza-Moquillaza R, Loza-Munarriz C, Rodríguez-Hurtado $D$, Arellano-Sacramento C, Hermoza-Moquillaza V. Automedicación en un distrito de Lima Metropolitana, Perú. Rev Medica Hered. 2016;27(1):15-21. doi:10.20453/RMH.V27।1.2779

7. RECOVERY Collaborative Group, Horby P, Lim WS, Emberson $\mathrm{JR}$, Mafham M, Bell JL, et al. Dexamethasone in Hospitalized Patients with Covid-19 - Preliminary Report. N Engl J Med. 2020. doi:10.1056/NEJMOA2021436

8. Filho PS da PS, Costa REAR da, Andrade IA da S, Sousa FW dos S, Júnior J de SA, Neto ASC, et al. Riscos da automedicação em idosos acometidos pelo coronavírus e outras síndromes respiratórias. Res Soc Dev. 2020;9(7):e458974211-e458974211. doi:10.33448/RSDv917.4211

9. Jamhour A, El-Kheir A, Salameh P, Hanna PA, Mansour H. Antibiotic knowledge and self-medication practices in a developing country: A cross-sectional study. Am J Infect Control. 2017;45(4):384-8. doi:10.1016/J.AJIC.2016.11.026

10. Montastruc J-L, Bondon-Guitton E, Abadie D, Lacroix I, Berreni A, Pugnet $G$, et al. Pharmacovigilance, risks and adverse effects of self-medication. Therapies. 2016;71(2):257-62. doi:10.1016/J. THERAP.2016.02.012 